\title{
Composition, identity and plural ontology
}

\author{
Roberto Loss $^{1}$ (D) \\ Received: 27 August 2019 / Accepted: 12 March 2020 / Published online: 26 March 2020 \\ (c) The Author(s) 2020
}

\begin{abstract}
According to 'Strong Composition as Identity' (SCAI), if an entity is composed of a plurality of entities, it is identical to them. As it has been argued in the literature, SCAI appears to give rise to some serious problems which seem to suggest that SCAItheorists should take their plural quantifier to be governed by some 'weak' plural comprehension principle and, thus, 'exclude' some kinds of pluralities from their plural ontology. The aim of this paper is to argue that, contrary to what may appear at first sight, the assumption of a weak plural comprehension principle is perfectly compatible with plural logic and the common uses of plural quantification. As I aim to show, SCAI-theorists can simply claim that their theory must be understood as formulated by means of the most 'joint-carving' plural quantifier, thus leaving open the possibility of other, less joint-carving, 'unrestricted' plural quantifiers. In the final part of the paper I will also suggest that SCAI-theorists should not only allow for singular quantification over pluralities of entities, but also for plural quantification over 'super-pluralities' of entities.
\end{abstract}

Keywords Composition as identity · Mereological universalism · Plural logic · Fundamentality $\cdot$ Structure

\section{Introduction}

Let an entity $x$ be the fusion of a plurality $Y$ of entities just in case each of the $Y \mathrm{~s}$ is part of $x$ and every part of $x$ overlaps (that is, has a part in common with) at least one of the $Y \mathrm{~s}^{1}$ :

(Fusion) $x F u Y={ }_{d f} \forall z(z \prec Y \rightarrow z \leq x) \wedge \forall z(z \leq x \rightarrow \exists w(w \prec Y \wedge O w z))$

\footnotetext{
${ }^{1}$ For alternative definitions of mereological fusion see Hovda (2009) and Varzi (2016: section 4).

Roberto Loss

robertoloss@gmail.com

1 Philosophisches Seminar, University of Hamburg, Überseering 35, 22297 Hamburg, Germany
} 
According to Strong Composition as Identity (or 'SCAI') whenever an entity $x$ is the fusion of a plurality $Y$ of entities, $x$ is literally identical to the $Y \mathrm{~s}^{2,3}$ :

(SCAI) $\forall x \forall Y(x F u Y \rightarrow x=Y)$

SCAI-theorists accept Leibniz's law in its full generality. ${ }^{4}$ However, Leibniz's law seems to give rise to some serious problems for SCAI. Consider, for instance, the properties 'being one' and 'being many': the whole is one; yet the parts it fuses are many; so, how could they be identical? For this reason, some SCAI-theorists claim that the instantiation of these problematic properties must be thought of as being relative either to a certain way to count the entities in question, or to a certain way to conceptualize them. ${ }^{5}$ This family of views can be called count-relative SCAI. ${ }^{6}$ In this paper I will focus only on non-count-relative SCAI, that is, the view according to which (i) wholes are literally identical to the parts they fuse, and (ii) problematic properties like 'being one' and 'being many' are absolutely instantiated, and not relative to a 'count' or to a 'way of conceptualizing'. ${ }^{7}$ Most importantly, non-count-relative SCAI accepts the following principle concerning the 'one-of' predicate which I will, thus, take to be valid throughout the paper:

(One-of) $\forall X \forall Y(X=Y \rightarrow \forall z(z \prec X \leftrightarrow z \prec Y))$

The main motivation behind SCAI is its apparent ability to give a straightforward account of the innocence of mereology. In fact, if a whole is identical to the parts it fuses, then insofar as one is committed to the existence of the parts, a commitment to the whole appears to come at no additional 'ontological cost' ${ }^{8}$ As it has been argued in the literature, however, non-count-relative SCAI (henceforth, simply 'SCAI') gives rise to some serious problems, since it appears to entail the so-called 'Collapse' principle, which says that, if an entity $x$ fuses a plurality $Y$ of entities, then something is a part

${ }^{2}$ See Cotnoir (2014) for an introduction to Composition as Identity and the distinction between 'strong' and 'moderate' Composition as Identity.

${ }^{3}$ In what follows (i) ' $x$ ', ' $y$ ', .., ' $z$ ' are singular variables, (ii) ' $X$ ', ' $Y$ ', .., ' $Z$ ' are plural variables, (iii) ' $y \prec X$ ' stands for ' $y$ is one of the $X \mathrm{~s}$ ', (iv) ' $x \leq y$ ' stands for ' $x$ is part of $y$ ', (v) 'Oxy' stands for ' $x$ overlaps with $y^{\prime}(O x y=d f \exists z(z \leq x \wedge z \leq y))$, and (vi) ' $x<y$ ' stands for ' $x$ is a proper part of $y$ ' $\left(x<y={ }_{d f} x \leq y \wedge x \neq y\right)$.

${ }^{4}$ Letting ' $\alpha$ ' and ' $\beta$ ' be schematic variables that can be replaced by either singular or plural variables, Leibniz's law can be formulated as follows (cf. Bricker 2019, p. 4):

(LL) $\forall \alpha \forall \beta(\alpha=\beta \rightarrow(\varphi(\alpha) \leftrightarrow \varphi(\beta))$

As Sider says 'Whatever else one thinks about identity, Leibniz's law must play a central role. [...] To deny it would arouse suspicion that their use of 'is identical to' does not really express identity' (Sider 2007, pp. 56-57).

5 For instance, according to Bøhn (2014), 'I can hold one and the same thing in my hand and truly say of it that it is one deck of cards, but fifty-two cards. One and the same thing is thus 1 when thought of under (or picked out by) the concept DECK OF CARDS, but 52 when thought of under (or picked out by) the concept CARD' (Bøhn 2014, p. 145).

${ }^{6}$ See, for instance, Bøhn (2014, 2019), Wallace (2011a, b), and Cotnoir (2013).

7 See, for instance, Payton (2019) and Loss (2019) on two recent ways of accounting for the idea that a whole is both one and many without relativizing 'being one' and 'being many' to counts or ways of conceptualizing.

8 On the idea that CAI entails that mereology is innocent see, among others, Cotnoir (2014, p. 7), Bennett (2015, p. 256), Varzi (2014, p. 49), and Hawley (2014, p. 72). 
of $x$ if and only if it is one of the $Y$ s (or, more simply, according to which an entity can fuse only the plurality of its parts) ${ }^{9}$ :

(Collapse) $\forall x \forall Y(x F u Y \rightarrow \forall z(z \leq x \leftrightarrow z \prec Y))$

As Sider (2014) and I (Loss 2019) have argued, the culprit behind these problems seems to be the standard plural comprehension principle, according to which if there is at least something that $\phi \mathrm{s}$, then there is the plurality of the $\phi$-ers:

(CMP) $\exists x \phi x \rightarrow \exists Y \forall x(x \prec Y \leftrightarrow \phi x)$

Therefore, both Sider (2014) and I (Loss 2019) have suggested that SCAI-theorists should instead employ some 'weak' plural comprehension principle entailing, as Sider (2014) puts it, that there are 'fewer pluralities than one normally expects' (Sider 2014, p. 213). In particular, I have argued that the best plural comprehension principle for SCAI-theorists is one that entails, jointly with SCAI and the assumption of atomism, that there are only pluralities of mereological atoms (Loss 2019).

The strategy of solving the problems of SCAI by embracing a weak plural comprehension principle may appear to be deeply problematic, at least prima facie. In fact, the kind of 'restriction' of the standard plural comprehension principle necessary to avoid all the bad consequences of Collapse appears to be so drastic that it makes plural logic and plural quantification useless for their common purposes such as: the interpretation of second-order logic, the foundation of set theory, or the semantic interpretation of plurals in natural languages. The aim of this paper is to argue that this line of thought can be resisted. As I will argue, SCAI-theorists can simply claim that the plural quantifier by means of which they express their theory is the most 'joint-carving' plural quantifier, in the sense of Sider (2011). This, however, appears to be perfectly compatible with the existence of a less-than-maximally-joint-carving plural quantifier obeying the usual, 'unrestricted' plural comprehension principle. I will thus conclude that the kind of ontologically innocent mereology promised by SCAI-theorists is not incompatible with plural logic. In the final part of the paper I will also address a couple of objections to this way of understanding SCAI and suggest that, in the same way in which SCAI allows for singular quantification over pluralities of entities (that is, for singular quantifications over things that are identical to pluralities of entities), it should also allow for plural quantification over pluralities of pluralities of entities, or 'super-pluralities' (that is, for plural quantifications over pluralities that have proper pluralities as 'members'). In what follows I will work against the backdrop of (i) classical mereology, axiomatized by means of the following three principles ${ }^{10}$ :

$\begin{array}{ll}\text { (Transitivity) } & \forall x \forall y \forall z((x \leq y \wedge y \leq z) \rightarrow x \leq z) \\ \text { (Weak Supplementation) } & \forall x \forall y(x<y \rightarrow \exists z(z \leq y \wedge \sim O z x)) \\ \text { (Universalism) } & \forall X \exists y(y F u X)\end{array}$

and (ii) atomism, thought of as the idea that every entity has atomic parts (' $A x$ ' stands for ' $x$ is a mereological atom' and is short for ' $\left.\sim \exists y(y<x)^{\prime}\right)^{11}$ :

(Atomism) $\forall x \exists y(y \leq x \wedge A x)$

\footnotetext{
9 See Sider (2007, 2014), and Yi $(1999,2014)$.

10 See Hovda (2009) for other ways to axiomatize classical mereology.

11 For a recent discussion of this definition of atomism see Varzi (2017).
} 


\section{How to avoid the bad consequences of Collapse}

The following plural comprehension principle is usually assumed to be an axiom of plural logic ${ }^{12}$ :

(CMP) $\exists x \phi x \rightarrow \exists Y \forall x(x \prec Y \leftrightarrow \phi x)$

(CMP) can be used to prove the 'Covering' principle, according to which, if $x$ is part of $y$, there is some plurality of entities $W$ such that $y$ fuses the $W$ s and $x$ is one of the $W \mathrm{~s}^{13}$ :

(Covering) $\forall x \forall y(x \leq y \rightarrow \exists W(y F u W \wedge x \prec W))$

In turn, SCAI and Covering entail the infamous 'Collapse' principle, according to which, if $x$ fuses a plurality $Y$ of entities, then something is a part of $x$ if and only if it is one of the $Y \mathrm{~s}$ (or, more simply, according to which an entity can fuse only the plurality of its parts) ${ }^{14,15}$ :

(Collapse) $\forall x \forall Y(x F u Y \rightarrow \forall z(z \leq x \leftrightarrow z \prec Y))$

As it has been shown in the literature, in the presence of the comprehension principle (CMP) the Collapse principle gives rise to some serious problems. ${ }^{16}$ Consider, for instance, the problem that I have called the 'Walls, Bricks, and Atoms' problem, or 'WaBrA' problem for short (Loss 2019: section 3). Suppose that a certain wall and a certain plurality of bricks are such that every brick is part of the wall and every part of the wall overlaps at least one of the bricks. By the definition of fusion, the wall fuses the plurality of the bricks. However, the wall has also other parts beside the bricks (like, for instance, all of its atomic parts) contra Collapse. Furthermore, Collapse has also been argued to entail mereological nihilism, that is, the claim that nothing has proper parts. ${ }^{17}$ The simplest argument to this effect goes as follows. Take any composite entity $x$. By (CMP) there is the plurality $Y$ of entities that are identical to $x . x$ clearly fuses the $Y$ s. It follows from Collapse that every part of $x$ is one of the $Y \mathrm{~s}$, and therefore, that every part of $x$ is identical to $x$, so that $x$ has no proper parts. Contradiction! Therefore, nothing has proper parts. ${ }^{18}$

\footnotetext{
12 See Linnebo (2017: section 1.2). I am also assuming in this paper that no plurality is empty (Ibid.) (NEP) $\forall X \exists y(y \prec X)$
}

13 Proof Suppose that, for some $x$ and $y, x$ is part of $y$. It follows that that there is something that is identical to either $x$ or $y$. By (CMP) we have, thus, that there is a plurality $W$ of entities such that something is one of the $W \mathrm{~s}$ if and only if it is identical to either $x$ or $y$. Therefore, $x$ is one of the $W \mathrm{~s}$. From the definition of fusion it follows that $y$ fuses the $W$ s.

14 Proof Suppose that $x$ fuses the $Y \mathrm{~s}$. By the definition of fusion we have that if an entity $z$ is one of the $Y \mathrm{~s}$, then $z$ is part of $x$. Conversely, if $z$ is part of $x$, it follows from Covering that there is some plurality $W$ such that $x$ fuses the $W \mathrm{~s}$ and $z$ is one of the $W \mathrm{~s}$. By SCAI, $x$ is identical to both the $W \mathrm{~s}$ and the $Y \mathrm{~s}$. Therefore, the $W \mathrm{~s}$ and the $Y$ s are the same plurality of entities. By (One-of) the $W \mathrm{~s}$ and the $Y$ s have the same members, so that $z$ is also one of the $Y$ s.

15 See Sider (2007, 2014), and Yi (1999, 2014). Here I am following in particular Sider (2014).

16 On the problems deriving from the Collapse principle see, among others, Sider (2007, 2014), Yi (1999, 2014), Calosi (2016), and Loss (2018, 2019).

17 See Calosi (2016) and Loss (2018).

18 See Loss (2018, p. 371). Gruszczyński (2015) presents a similar argument using sets instead of pluralities. 
Following Sider (2014), one can take the culprit behind these problems to be (CMP). In fact, on the one hand, (CMP) appears to be necessary to prove Collapse. ${ }^{19}$ On the other hand, it is (CMP) which (implicitly) allows us to infer from the fact that something is a brick that there is the plurality of the bricks, and from the fact that $x$ exists that there is the plurality of the entities that are identical to $x$. Therefore, the moral of the story in this case seems to be that SCAI-theorists must reject (CMP) and embrace a weaker comprehension principle. Both Sider (2014) and I (Loss 2019) discuss alternative plural comprehension principles on behalf of SCAI-theorists which are formulated by means of the notion of schematic fusion (or 'S-fusion'):

(S-Fusion) $x S F u \phi={ }_{d f} \forall z(\phi z \rightarrow z \leq x) \wedge \forall z(z \leq x \rightarrow \exists w(\phi w \wedge O z w))$

According to this definition, an entity $x$ is the S-fusion of everything that $\phi \mathrm{s}$ if and only if, every $\phi$-er is a part of $x$ and every part of $x$ overlaps at least some $\phi$-er. ${ }^{20}$ Both Sider (2014) and I (Loss 2019) also assume the principle of Unrestricted Schematic Fusion

(USF) $\exists y \phi y \rightarrow \exists y(y S F u \phi)$

and formulate our 'Weak', 'Properly Weak' and 'Atomic' comprehension principles as follows (' $\phi[x, y]$ ' is short for ' $\left.x \neq y \wedge \phi x \wedge \phi y^{\prime}\right)^{21}$

(WCP) $\exists x \phi x \rightarrow \exists x \exists Y(x S F u \phi \wedge \forall z(z \prec Y \leftrightarrow z \leq x))$

(PWCP) $\exists x \exists y \phi[x, y] \rightarrow \exists z \exists Y(z S F u \phi \wedge \forall x(x \prec Y \leftrightarrow x<z))$

(ACP) $\quad \exists x \exists y \phi[x, y] \rightarrow \exists z \exists Y(z S F u \phi \wedge \forall x(x \prec Y \leftrightarrow(x<z \wedge A x)))$

As I show in detail (Loss 2019, pp. 7-12), (WCP), (PWCP) and (ACP) all appear to successfully ward off both the WaBrA problem and the threat of mereological nihilism. In fact, on the one hand, the WaBrA problem depends on the possibility that a composite entity fuse different pluralities of entities. However, it is easy to check that (WCP), (PWCP) and (ACP) all entail that a composite entity can fuse at most one plurality of entities: the plurality of its parts, according to (WCP); the plurality of its proper parts, according to (PWCP); the plurality of its atomic parts, according to (ACP). On the other hand, the threat of nihilism stemming from Collapse appears to depend on the possibility of what I call 'incomplete thick pluralities' (Loss 2019, p. 5), that is, pluralities of entities containing all the parts of a certain entity except for

\footnotetext{
19 (CMP) is needed to prove the Covering principle. In fact, without (CMP) there is no guarantee that, if $x$ is part of $y$, then there is some plurality of entities containing $x$ and such that $y$ is their fusion (see above).

20 Contrary to (Fusion), (S-fusion) doesn't employ plural quantification. Therefore, if the plural quantifier is governed by some 'weak' comprehension principle, from the fact that there is the S-fusion of (say) ' $x$ is red', it doesn't necessarily follow that there is the fusion of the plurality of red things (see also below).

21 My original formulations of (PWCP) and (ACP) featured only ' $\exists x \phi x$ ' in the antecedent (Loss 2019, p. 11). However, this is clearly inconsistent with cases in which there is only one atomic $\phi$-er (under the assumption that there are no empty pluralities; see footnote 12). Furthermore, if (PWCP) and (ACP) are reformulated with ' $\exists x \exists y \phi[x, y]$ ' in their antecedent, then (even assuming SCAI) they appear to be both compatible with the existence of improper pluralities of atoms. Therefore, the theories that I call 'CAI + (PWCP)' and 'ACAI' (Loss 2019) must be understood as featuring the present formulations of (PWCP) and (ACP) as well as the following additional axiom, ruling out the existence of improper pluralities of atoms:
}

(NIPA) $\forall X \exists y \exists z(A y \wedge A z \wedge y \neq z \wedge y \prec X \wedge z \prec X)$

(see also Loss 2020). 
some of its proper parts (like the 'improper' plurality of the entities identical to some composite entity $x$, or the plurality of entities identical to either $x$ or one of its proper parts $y$ ). However, (WCP), (PWCP) and (ACP) all provably entail that there are no incomplete thick pluralities. $^{22}$

It may seem, therefore, that equipped with either (WCP), (PWCP), or (ACP) SCAI is a theory that is at least safe from some of the most serious objections that have been levelled against it. It can be objected, however, that this is but a Pyrrhic victory for SCAI-theorists, as (WCP), (PWCP), and (ACP) all appear to be so weak that they make plural logic useless for most of its common purposes, such as: the interpretation of second-order logic, the foundation of set theory, or the semantic interpretation of plurals in natural languages. ${ }^{23}$ For instance, in the case of the wall and the bricks, it follows from (WCP), (PWCP), and (ACP) that there is no plurality that is the plurality of 'the bricks' (that is, the plurality $Y$ such that something is one of the $Y$ s if and only if it is a brick). This means that, there is no plurality available to stand for the property of being a brick, the set of bricks, or indeed for the English phrase 'the bricks'. (WCP), (PWCP), and (ACP) are, thus, incompatible with the very examples that are put forward in the literature to motivate SCAI, like the idea that a piece of land divided in six parcels is identical to the six parcels taken together, or the idea that a six-pack of orange juice is identical to the six cans of orange juice taken together (Baxter 1988). ${ }^{24}$ It may seem, therefore, that the conclusion one should draw from the arguments put forward by Sider (2014) and me (Loss 2019) is that SCAI ought to be simply rejected as incompatible with plural logic. ${ }^{25}$

As I will argue in what follows, this line of reasoning can be resisted.

\section{The most joint-carving plural quantifier}

According to Sider (2011), 'metaphysics, at the bottom, is about the fundamental structure of reality' (Sider 2011, p. 1), where the idea that reality has a fundamental structure means that there is a 'privileged description' of the world- 'an objectively correct way to "write the book of the world" (Sider 2011, p. i). In the same way in

\footnotetext{
22 As for (WCP), see Loss (2019, p. 10). As for (PWCP) and (ACP), suppose that $X$ is an incomplete thick plurality and that $x$ is the member of the $X \mathrm{~s}$ that has each of the $X \mathrm{~s}$ as parts. Let $\phi$ be ' $v$ is part of $x$ '. The $X s$ are an incomplete thick plurality of entities. Therefore, $x$ is a composite entity, so that there are in this case at least two $\phi$-ers (actually, at least three, given Weak Supplementation). It follows from both (PWCP) and (ACP) that there is a plurality $Y$ of entities that is the plurality of either all the proper parts or all the atomic parts of the S-fusion of everything that $\phi \mathrm{s}$. In both cases we have that $x$ fuses both the $X \mathrm{~s}$ and the $Y \mathrm{~s}$ so that the $X \mathrm{~s}$ and the $Y s$ must be the same plurality. However, $x$ is one of the $X \mathrm{~s}$ but not one of the $Y \mathrm{~s}$. Contradiction! Therefore, there are no incomplete thick pluralities.

23 See, among many others, Boolos (1984, 1985), McKay (2006), and Moltmann (2016).

24 Jointly with SCAI the principles (WCP), (PWCP), and (ACP) can in fact all be used to prove that there is no plurality that is the plurality of 'the parcels of land', or the plurality of 'the cans in the six-pack'.

25 'The list of applications for plural quantification grows by the year. [...] all would be undermined by strong composition as identity' (Sider 2007, p. 66). 'My reason for rejecting strong composition as identity is essentially the same as that of Sider in 'Parthood': that it is incompatible with the framework of plural logic' (Bricker 2016, p. 280). Notice, however, that Sider (2014) doesn't find his argument from plural logic convincing anymore (see Sider 2014, p. 211, footnote 2; see also below, Sect. 3, and especially footnote $38)$.
} 
which predicates like 'blue' and 'green' can intuitively be said to be more joint-carving than 'grue' and 'schmue', for Sider (2011) every expression of a language can be said to be more or less joint-carving and, thus, more or less 'metaphysically perspicuous' in this sense: logical connectives, quantifiers, tense and modal operators, et cetera. For instance, Sider embraces ontological realism, understood as the view that there is a perfectly joint-carving singular existential quantifier. However, as he stresses, this is not incompatible with the existence of other, less joint-carving existential quantifiers (like natural language quantifiers, for instance):

Suppose that, fundamentally, there are very few things. Suppose [...], for example, that there exist, in the fundamental sense, nothing but subatomic particles. Given such a sparse ontology, the most plausible view about natural language quantifiers might be that they do not carve at the joints. [...] So even if there is $a$ joint-carving sort of quantification, the quantifiers of ordinary language might not carve at the joints. (Sider 2011, p. 171)

As Sider (2014) and I (Loss 2019) have argued there appear to be good reasons to take the plural quantifier used by SCAI-theorists to be governed by a comprehension principle that is weaker than (CMP). However, as the existence of a perfectly jointcarving singular quantifier ranging (say) only over subatomic particles doesn't rule out the existence of other, less joint-carving, singular quantifiers ranging also over tables and chairs, also the existence of a plural quantifier governed by a comprehension principle weaker than (CMP) doesn't appear to rule out the existence of other plural quantifiers for which (CMP) is valid. ${ }^{26}$

An important question concerning the relation between quantification and the fundamental structure of reality is whether there are any perfectly joint-carving plural quantifiers. Should we take the 'book of the world' to be written also by means of plural quantifiers, or should we instead agree with Sider (2011) that, while 'there is no significant pressure here to admit metaphysically fundamental plural quantification' (p. 212), the 'addition of second-order quantifiers to fundamental ideology immensely increases the complexity of one's fundamental theory' (p. 209)? Here we don't need to take a stand on how this issue may be resolved. In fact, whether or not there is any plural quantifier that is perfectly joint-carving, ${ }^{27}$ SCAI-theorists can simply claim (i) that their theory must be understood as employing the most joint-carving plural quantifier, and (ii) that there are other, less joint-carving plural quantifiers which are governed by (CMP) and that can, thus, be adopted for the common applications of plural logic.

I have called 'Atomic Composition as Identity' (or 'ACAI' for short) the version of SCAI which assumes (ACP) as its plural comprehension principle, entailing, thus, that every composite entity is identical to the plurality of atoms it fuses (Loss 2019). Let us assume in what follows that ACAI is the relevant instance of SCAI. ACAI entails that the plural quantifier by which it is formulated quantifies only over pluralities of

\footnotetext{
${ }^{26}$ Notice, however, that (as I make clear below) in this case there is a version of (CMP) that $i s$ valid also for the most joint-carving plural quantifier, and namely, the one featuring also the most joint-carving singular quantifier.

27 See Sider (2011, pp. 208-215) for some reasons not to accept the idea that higher-order quantification is perfectly joint-carving.
} 
atoms. ${ }^{28}$ ACAI-theorists may, thus, embrace a metaontology inspired by Sider (2011) and justify the weakness of (ACP) as follows:

(A1) There is one singular existential quantifier which is perfectly joint-carving;

(A2) The most joint-carving singular existential quantifier quantifies only over mereologically atomic entities, ${ }^{29}$

(A3) The most joint-carving plural quantifier quantifies over all and only pluralities of entities that are quantified over by the most joint-carving singular quantifier;

(A4) Therefore, the most joint-carving plural quantifier must be thought of as being governed by a comprehension principle weaker than (CMP) and along the lines of (ACP)

(A1) and (A2) are both perfectly legitimate metaphysical positions (even if not completely uncontroversial $)^{30}$ that appear to be perfectly in keeping with ACAI. (A3) has at least the ring of plausibility to it. Quite intuitively, in fact, a plurality of fundamental entities $^{31}$ strikes one as being more joint-carving (more natural, less gerrymandered) than a plurality comprising both fundamental and non-fundamental entities. At the same time, it is difficult to see what could possibly justify the idea that only plural quantification over the latter kind of pluralities is possible, and, thus, that the mostjoint carving plural existential quantifier does not manage to quantify over pluralities of only fundamental entities.

According to this way of justifying the weakness of (ACP), the central claim of SCAI (according to which every entity that is the fusion of a plurality of entities is identical to them) should be thought of as being formulated by means of a non-

${ }^{28}$ Consider an arbitrary plurality $X$ of entities. ACAI entails that there are no incomplete thick pluralities (see footnote 21). Therefore, either the $X \mathrm{~s}$ are an improper plurality containing only one atom or they are a proper plurality of entities. Suppose that the $X \mathrm{~s}$ are a proper plurality of entities. By Universalism, there is an entity $y$ that is the fusion of the $X \mathrm{~s}$. By (SCAI) $y$ is identical to the $X$ s. Let $\phi$ be ' $v$ is one of the $X$ s'. Since $X$ is a proper plurality, there are at least two $\phi$-ers in this case, so that it follows from (ACP) that there is an entity $z$ and a plurality $Y$ such that $z$ is the S-fusion of everything that is one of the $X$ s and something is one of the $Y \mathrm{~s}$ if and only if it is an atomic proper part of $z$. It follows from the definition of fusion that $z$ fuses both the $Y \mathrm{~s}$ and the $X \mathrm{~s}$. By (SCAI), $z$ is identical to both of them. Therefore, the $Y \mathrm{~s}$ and the $X \mathrm{~s}$ are the same plurality. By (One-of) it follows that something is one of the $X \mathrm{~s}$ if and only if it is an atomic part of $y$. The $X \mathrm{~s}$ are, thus, a plurality of atoms.

${ }^{29}$ One way to formulate the idea that the most joint-carving singular existential quantifier quantifies only over mereologically atomic entities may be that of saying that there is no true sentence of the form ' $\exists x \exists y(y<x)$ ' such that its outmost quantifier ' $\exists x$ ' is the perfectly joint-carving singular existential quantifier.

30 'Quantifier variantists' may object to (A1) by claiming that no quantifier is perfectly joint-carving, while 'ontological pluralists' may instead object that more than one quantifier is perfectly joint-carving (see Sider 2011, pp. 175-180, and Javier-Castellanos 2019 on this understanding of quantifier variantism and ontological pluralism, respectively). Instead, (A2) may be rejected by priority monists à la Schaffer (2010).

31 By 'fundamental entity' I mean an entity that is quantified over by the perfectly joint-carving singular quantifier. Therefore, an entity is fundamental in this sense if and only if its existence is fundamental, while its nature might still be derivative. For instance, according to Sider, '[for a] mereological universalist like David Lewis [... the] existence of tables and chairs is just as fundamental as the existence of electrons (in contrast, perhaps, with smirks and shadows, which do not exist fundamentally). However, tables and chairs have nonfundamental natures' (Sider 2011, p. 165). Therefore, a plurality of fundamental entities in this sense is a plurality of entities such that if something is one of them, then it exists in the fundamental sense. 
perfectly-joint-carving singular quantifier (capable of ranging also over composite entities) and the most joint-carving plural quantifier (ranging only over pluralities of atoms). ${ }^{32}$ Thus understood, SCAI has a kind of 'reductive' flavour to it, so to speak, as it identifies composite, metaphysically derivative entities with their most fundamental plural and atomic base. ${ }^{33}$ What is most important, however, is that all this is clearly compatible with the existence of some less joint-carving plural quantifier governed by the 'unrestricted' plural comprehension principle (CMP) and quantifying also over pluralities of entities not containing only fundamental atoms. Therefore, in the very same way in which Sider (2011) claims that, although the most joint-carving singular quantifier doesn't quantify over tables, the ordinary English quantifier does, ACAItheorists can claim that, although their plural quantifier only quantifies over pluralities of atoms, there is another less joint-carving plural quantifier that is governed by (CMP) and that can, thus, be used for all the other common applications of plural logic. In this sense, although it is true that, fundamentally speaking, there is, for instance, no plurality that is the plurality of the bricks composing the wall (but only the plurality of the atomic parts of the wall), it is nevertheless also true that some plurality of entities are such that something is one of them if and only if it is a brick. It's just that in the latter case we are not employing the most fundamental plural quantifier, but instead an 'unrestricted', less fundamental one. Notice, furthermore, that it also follows from what has been said thus far that there is a sense in which (CMP) is true also for the most joint-carving plural quantifier employed by ACAI-theorists, namely, when (CMP) is formulated also by means of perfectly joint-carving singular quantifiers (' $\mathcal{E}$ ' and ' $\mathcal{A}$ ' stand here for the perfectly joint-carving singular and universal quantifier, respectively) ${ }^{34,35}$ :

\footnotetext{
32 Understood in this way, ACAI may qualify as a version of nihilism in Sider's (2013) sense, that is, as 'the view that in the fundamental sense, there are no composite entities' (Sider 2013, p. 253). Notice, however, that Sider's 'nihilism' is compatible with the idea that (using a non-perfectly-joint-carving singular quantifier) there is some composite entity.

33 Notice that this may be understood as a way of saying that atomic parts 'ground the whole and are identical to it' (Loss 2016).

${ }^{34}$ So formulated $\left(\mathrm{CMP}_{\mathrm{F}}\right)$ allows for the existence of improper pluralities of atoms. In order to ban those pluralities from ACAI's ontology $\left(\mathrm{CMP} 2_{\mathrm{F}}\right)$ and (NIPA) must be assumed instead:
}

$\left(\mathrm{CMP} 2_{\mathrm{F}}\right) \quad \mathcal{E} x \mathcal{E} y \phi[x, y] \rightarrow \exists Y \mathcal{A} x(x \prec Y \leftrightarrow \phi x)$

$\left(\mathrm{NIP}_{\mathrm{F}}\right) \quad \forall X \mathcal{E} y \mathcal{E} z(y \neq z \wedge y \prec X \wedge z \prec X)$

(see on this issue footnotes 21 and 41).

35 Objection Suppose a composite entity $b$ is identical to a certain plurality of atoms (the ' $a$ s'). According to this way of understanding ACAI, the most joint-carving plural quantifier quantifies over the $a$ s. How come, then, that the most joint-carving singular quantifier doesn't quantify over $b$ given that $b$ is identical to the $a$ s? How can the whole fail to exist in the joint-carving sense of 'exist', if the atomic parts to which it is identical do? (Many thanks to an anonymous referee for this Journal for pressing me on this question) Reply: Because representing the as as many is more joint-carving than representing them as one, so to speak. In other words, the 'portion of reality' corresponding to $b$ /the $a$ s is most perspicuously represented as being a plurality of atoms than as being a single entity. Notice, furthermore, that I am not assuming here that the most joint-carving plural quantifier is perfectly joint-carving, and thus, that it is as joint-carving as the most joint-carving singular quantifier. If the most joint-carving plural quantifier is not perfectly joint-carving, then a fortiori the fact that there are (in the most-yet-not-perfectly joint-carving sense) some entities to which $b$ is identical appears to give no reason to think that, therefore, there must be something (in the perfectly joint-carving sense) that is identical to $b$. 
$\left(\mathrm{CMP}_{\mathrm{F}}\right) \quad \mathcal{E} x \phi x \rightarrow \exists Y \mathcal{A} x(x \prec Y \leftrightarrow \phi x)$

In other words, the only version of (CMP) that is invalid is the one in which its singular quantifiers are the unrestricted, non-perfectly joint-carving ones, and its plural quantifier is the most joint-carving, restricted one. When both the singular and the plural quantifier used in formulating (CMP) are either unrestricted or the most jointcarving ones, then (CMP) is indeed valid.

Finally, while ACAI-theorists appear to be perfectly in position to justify the weakness of (ACP) by means of an argument like (A1)-(A4), things appear to be more complicated in the case of (WCP) and (PWCP). Let us focus on (WCP) [the reasoning being similar for (PWCP)]. Assuming that the world is endowed with enough mereological structure, (WCP) guarantees the existence of pluralities containing all the parts of some composite entity. Therefore, the counterpart of (A2) in this case would have to allow also for all the parts of some composite entity to be fundamental-a claim that sounds counter-intuitive, at least prima facie. Furthermore, as I have shown (Loss 2019, pp. 12-14), given (WCP) and SCAI the plural quantifier is not guaranteed to be well-behaved. For instance, (WCP) and SCAI taken together do not guarantee that any two pluralities of entities have a plural union. ${ }^{36}$ However, if two pluralities are quantified over by the fundamental quantifier, then-at least on the background of the plausible principle (A3) — the entities belonging to both of them are fundamental entities. So, why shouldn't the most fundamental plural quantifier be able to plurally quantify over their plural union? It seems, thus, to be more difficult to explain the weakness of (WCP) or (PWCP) in a way that is similar to (A1)-(A4). If this is correct, we may have some additional reason to believe that, as I have suggested (Loss 2019), ACAI is indeed the best version of SCAI. ${ }^{37,38}$

\footnotetext{
36 Given three atoms $a, b$, and $c$, (WCP) + SCAI entails that there is the plurality of all the parts of $a+$ $b$ and the plurality of all the parts of $b+c$, even if there is no plurality containing only the things that are either part of $a+b$ or $b+c$ (the smallest plurality containing these two pluralities is the plurality of all the parts of $a+b+\mathrm{c}$, which also contains $a+c$ and $a+b+c$ ).

37 It may be suggested that (WCP) + SCAI-theorists can claim that their quantifier, although less gerrymandered than the quantifier that is governed by $(\mathrm{P} 2)$ is not the most joint-carving plural quantifier. In this case, however, at least in the presence of (A1)-(A3) ACAI ought to be clearly preferred as the best version of SCAI, connecting every composite entity to its most fundamental plural base.

38 Notice, finally, that what I have argued in this section may be seen as just a way of articulating and making explicit the following remark of Sider: 'I rejected [in Sider (2007)] (fun, interesting) composition as identity [on the basis that it 'alters Boolos's logic of plural quantification in various ways' (Sider 2014, p. 211)], but I no longer find that argument convincing since I now doubt that Boolos's logic should be taken as metaphysically basic' (Sider 2014, p. 211, fn 2). See also Sider (2011, section 9.15).
} 


\section{SCAI, improper pluralities and pluralities of pluralities}

Let ' $\Sigma$ ' and ' $\Pi$ ' stand for the 'generic', non-joint-carving plural existential and universal quantifiers governed by (CMP), ' $x x$ ', ' $y y$ ', ' $z z$ ', $\ldots$ be 'generic' plural variables, and ' $\sqsubset$ ' stand for the generic 'one of' relation. Let's also keep assuming ACAI as the relevant version of SCAI. ' $\Sigma$ ' and ' $\Pi$ ' also range over pluralities of atoms. Therefore, ACAI entails in this case the following conditional claim (where ' $\mathbb{A} y y$ ' stands for 'the $y y$ are a plurality of atoms' and is short for ' $\forall x(x \prec y y \rightarrow A x)$ '; ' $x F y y$ ' stands for ' $x$ is a mereological fusion of the $y y$ '):

\section{$\left(\mathrm{ACAI}_{\mathrm{C}}\right) \quad \forall x \Pi y y((\mathbb{A} y y \wedge x F y y) \rightarrow x=y y)$}

According to $\left(\mathrm{ACAI}_{\mathrm{C}}\right)$, if an entity $x$ fuses a plurality of atoms, then it is literally identical to them. By using these 'generic' plural quantifiers we can, thus, say that, although the wall fuses the bricks of which it is made, it is nevertheless not identical to them. The only plurality of entities the wall is identical to are its 'atomic footprint' (Loss 2019, p. 15), that is, the plural union of all the pluralities of entities that are identical to one of the bricks composing the wall. It is, therefore, this fact that accounts for the intuition that the wall is 'nothing over and above' the plurality of its bricks (the piece of land is nothing over and above its parcels; the six-pack is nothing over and above the six cans of beer, et cetera; more on this below).

One may object as follows. The generic plural quantifier is thought of as being governed by (CMP) in this sense:

$\left(\mathrm{CMP}_{\mathrm{G}}\right) \quad \exists x \phi x \rightarrow \Sigma y y \forall x(x \sqsubset y y \leftrightarrow \phi x)$

$\left(\mathrm{CMP}_{\mathrm{G}}\right)$ entails that there is the improper (generic) plurality having the wall as its only member, (that is, the plurality of the things that are identical to the wall). However, quite independently of SCAI, it appears highly plausible to take an improper plurality of entities to be identical to its only member. After all, contrary to sets, pluralities of entities just are their members: there is nothing 'more' to a plurality than its members. It follows, thus, that the wall is identical to both (what we may call) its improper generic plurality and the plurality of its atoms. However, this cannot be the case given the generic version of (One-of) (see Sect. 1):

$\left(\right.$ One-of $\left._{\mathrm{G}}\right) \quad \Pi x x \Pi y y(x x=y y \rightarrow \forall z(z \sqsubset x x \leftrightarrow z \sqsubset y y))$

In fact, both the plurality of atomic parts of the wall and the (improper) plurality of things that are identical to the wall are quantified over by the generic plural quantifier:

(4) $\sum x x \sum y y(\forall z(z \sqsubset x x \leftrightarrow(A z \wedge z \leq w)) \wedge \forall z(z \sqsubset y y \leftrightarrow z=w))$

Therefore, in the presence of $\left(\right.$ One-of $\left._{\mathrm{G}}\right)$, they cannot be identical, given that they have different members.

What this objection shows is that there is also one kind of generic pluralities whose existence should be rejected by ACAI-theorists, namely, improper generic pluralities. ACAI-theorists should, in other words (i) embrace $\left(\mathrm{CMP}_{\mathrm{G}} *\right)$ instead of $\left(\mathrm{CMP}_{\mathrm{G}}\right)$ as the comprehension principle governing their generic plural quantifier, and (ii) add to their theory an axiom banning improper pluralities from their plural ontology:

$\left(\mathrm{CMP}_{\mathrm{G}} *\right) \quad \exists x \exists y \phi[x, y] \rightarrow \Sigma y y \forall x(x \sqsubset y y \leftrightarrow \phi x)$ 
$\left(\mathrm{NIPA}_{\mathrm{G}}\right) \quad \Pi x x \exists y \exists z(y \neq z \wedge y \prec x x \wedge z \prec x x)$

Notice, however, that this 'restriction' of $\left(\mathrm{CMP}_{\mathrm{G}}\right)$ appears to be harmless, as the pluralities that in this case are 'missing' from the plural ontology of ACAI-theorists, so to speak, are still quantified over by their singular quantifier. For instance, according to $\left(\mathrm{NIPA}_{\mathrm{G}}\right)$, there is no plurality $x x$ such that something is one of the $x x$ if and only if it is identical to the wall. However, if such a plurality existed it would be identical to the wall. Yet the wall is still quantified over by the generic singular quantifier of ACAI. Therefore, ACAI-theorists can simply invoke the wall itself anytime the improper plurality of the wall' is needed by some of the applications of plural logic and plural quantification.

A second important objection to consider is the following one. According to this picture, there is indeed a plurality $b b$ of entities (namely, the plurality of the bricks) that are such that a certain brick $b$ is one of them:

(4) $\Sigma x x(x x=b b \wedge b \sqsubset b b)$

At the same time, there is also a certain plurality $a a$ of atoms such that $b$ is identical to them:

(5) $\Sigma x x(x x=a a \wedge b=a a)$

We have, thus, that $b$ is not only one of the $b b$ but also identical to the $a a$ :

(6) $b \sqsubset b b \wedge b=a a$

From (6) it follows from Leibniz's law (which ACAI-theorists, qua SCAI-theorists, accept in its full generality) that the $a a$ - the atoms to which $b$ is identical-are also one of the $b b$ - the bricks (where, clearly, the $a a$ and the $b b$ are two different pluralities, given Leibniz's law)!

$$
a a \sqsubset b b \wedge a a \neq b b
$$

The idea that a plurality of entities may be 'one of' another plurality is not new in the literature on higher-order plural quantification. ${ }^{39}$ Notice, however, that strictly speaking (7) doesn't commit $\left(\mathrm{ACAI}_{\mathrm{C}}\right)$-theorists to resorting to higher-order plural quantifiers, but only to allowing their (generic) 'one-of' relation to admit of plural terms on its left-hand side. ACAI already entails that there is some non-perfectly-jointcarving existential quantifier that can singularly quantify over pluralities of entities, at least in the sense that it can singularly quantify over entities that are identical to (proper) pluralities of entities:

(8) $\exists x \exists Y(x=Y \wedge \exists y \exists z(y \neq z \wedge y \prec Y \wedge z \prec Y))$

Notice that it follows from (8) and Leibniz's law that-since $x$ is identical to the $Y \mathrm{~s}$ and there are two different entities such that each of them is one of the $Y_{\mathrm{s}}$-there is some $x$ and some $y$ such that $y$ is one of $x$ and $x$ is different from $y$ [by (One-of ${ }_{\mathrm{G}}$ )]:

(9) $\exists y \exists x(y \prec x \wedge x \neq y)$

\footnotetext{
39 See, for instance, Rayo (2006, p. 227). On super-plurals see also, among others, Hazen (1997), Linnebo and Nicolas (2008), Oliver and Smiley (2013, pp. 275-279), Simons (2016), and Linnebo (2017: 2.4).
} 
This may come as a surprise, as many authors appear to think that a singular entity can only be one of itself. ${ }^{40}$ However, following my suggestion (Loss 2019: pp. 15-17), ACAI-theorists can simply take the one-of relation to be a proper relation ('proper' as in 'proper parthood' and 'proper subset'), and claim that no entity can be (in this sense) one of itself. ${ }^{41}$ At the same time, ACAI-theorists can also insist that, if an entity $x$ is identical to a plurality $y y$ of entities, then, of course, if something is one of the $y y$ it is also one of $x$ (see Loss 2019: pp. 16-17). Therefore, ACAI-theorists can conclude that what (7) shows is only that, in a similar way in which the generic, non-perfectly-jointcarving existential quantifier can singularly quantify over pluralities of entities, so the generic, non-maximally-joint-carving plural quantifier can plurally quantify over (proper) $^{42}$ super-pluralities of entities, that is pluralities having (proper) pluralities as members (in what follows ' $\mathcal{P} x x$ ' stands for 'the $x x$ are a proper plurality of entities' and is short for ' $\left.\exists y \exists z(y \neq z \wedge y \sqsubset x x \wedge z \sqsubset x x)^{\prime}\right)$ ):

\section{(10) $\Sigma x x \Sigma y y \Sigma z z(\mathcal{P} x x \wedge \mathcal{P} y y \wedge x x \neq y y \wedge x x \sqsubset z z \wedge y y \sqsubset z z)$}

Once (8) and (9) are accepted as unproblematic, there appears to be nothing wrong in either (10) or (7).

The introduction of the following notation might help clarify things. Let ' $~ x x$. $\phi x x$ ' stand for the plural definite description 'the $x x$ that $\phi$ '43 and ' $\left[x_{1}, \ldots, x_{n}\right]$ ' be short for 'the plurality of things that are identical to either $x_{1}, \ldots$, or $x_{n}$ ':

$$
\text { (TheP) }\left[x_{1}, \ldots, x_{n}\right]=d f \iota x x . \forall z\left(z \sqsubset x x \leftrightarrow\left(z=x_{1} \vee \ldots \vee z=x_{n}\right)\right)
$$

Suppose, then, that there are just (i) the three mereological atoms $a_{1}, a_{2}$, and $a_{3}$, (ii) the three binary sums of atoms $b_{1}=a_{1}+a_{2}, b_{2}=a_{1}+a_{3}$, and $b_{3}=a_{2}+a_{3}$ (the 'bricks') and (iii) the fusion $c$ of all the atoms. Given $\left(\mathrm{ACAI}_{\mathrm{C}}\right)$ the following all hold:

$$
\begin{aligned}
& \text { (i) } b_{1}=\left[a_{1}, a_{2}\right] \\
& \text { (ii) } b_{2}=\left[a_{1}, a_{3}\right] \\
& \text { (iii) } b_{3}=\left[a_{2}, a_{3}\right]
\end{aligned}
$$

It follows from (TheP), (11) and (12) that the plurality of the bricks is identical to the plurality of $\left[a_{1}, a_{2}\right],\left[a_{1}, a_{3}\right]$, and $\left[a_{2}, a_{3}\right]$ taken together:

\footnotetext{
$\overline{40}$ See, for instance, Yi (1999, p. 146) and Calosi (2018, pp. 287-288).

41 In order to claim that no entity can be one of itself we must exclude the existence of improper pluralities of atoms [whose existence is not excluded by SCAI + (ACP)], and thus, assume as additional axiom either the principle (NIPA), according to which every plurality contains at least two atoms (see footnote 21), or more simply, the claim that every plurality is a proper plurality (that is, a plurality that contains at least two members):
}

(2M) $\forall X \exists x \exists y(x \prec X \wedge y \prec Y \wedge x \neq y)$

42 A proper super-plurality is a super-plurality containing more than one member. As it appears intuitive to take an improper plurality to be identical to its only member, so it also seems plausible to take an improper super-plurality to be identical to the only plurality it contains.

43 I assume here that plural definite descriptions are eliminable using Russell's theory of descriptions:

$$
\psi(\iota x x . \phi x x)={ }_{d f} \exists x x(\phi x x \wedge \forall y y(\phi y y \rightarrow y y=x x) \wedge \psi x x)
$$


(14) $\left[b_{1}, b_{2}, b_{3}\right]=\left[\left[a_{1}, a_{2}\right],\left[a_{1}, a_{3}\right],\left[a_{2}, a_{3}\right]\right]$

In fact, on the one hand we have that $\left[b_{1}, b_{2}, b_{3}\right]$ is the plurality of things that are identical to either $b_{1}, b_{2}$, or $b_{3}$ :

$$
\left[b_{1}, b_{2}, b_{3}\right]=\iota x x . \forall z\left(z \sqsubset x x \leftrightarrow\left(z=b_{1} \vee z=b_{2} \vee z=b_{3}\right)\right)
$$

on the other hand, from (11) we have that $b_{1}, b_{2}$, and $b_{3}$ are identical to $\left[a_{1}, a_{2}\right]$, $\left[a_{1}, a_{3}\right]$, and $\left[a_{2}, a_{3}\right]$, respectively:

(16) $b_{1}=\left[a_{1}, a_{2}\right] \wedge b_{2}=\left[a_{1}, a_{3}\right] \wedge b_{3}=\left[a_{2}, a_{3}\right]$

(14) can be simply derived by (15) and (16) by Leibniz's Law, which ACAI-theorists (qua SCAI-theorists) accept in its full generality. ${ }^{44}$ Therefore, (7) can be simply seen as belonging to the family of the prima facie odd claims $\left(\mathrm{ACAI}_{\mathrm{C}}\right)$ are committed to due to Leibniz's Law, like the claim [expressed by (14)] that $\left[b_{1}, b_{2}, b_{3}\right]$ are identical to [[ $\left.\left.a_{1}, a_{2}\right],\left[a_{1}, a_{3}\right],\left[a_{2}, a_{3}\right]\right]$, or the claim that, since $a_{1}$ is part of $b_{1}$ and $b_{1}$ is identical to $\left[a_{1}, a_{2}\right]$, then $a_{1}$ is not only one of $\left[a_{1}, a_{2}\right]$, but also part of $\left[a_{1}, a_{2}\right]$ :

$$
a_{1} \leq\left[a_{1}, a_{2}\right]
$$

All these results might seem startling at first. At a closer look, however, they shouldn't come as a surprise once $\left(\mathrm{ACAI}_{\mathrm{C}}\right)$ is accepted: if $\left[a_{1}, a_{2}\right]$ are identical to $b_{1}$, then, of course, $\left[a_{1}, a_{2}\right]$ are also one of $\left[b_{1}, b_{2}, b_{3}\right]$; if $b_{1}, b_{2}$, and $b_{3}$ are identical to $\left[a_{1}, a_{2}\right],\left[a_{1}, a_{3}\right]$, and $\left[a_{2}, a_{3}\right]$, respectively, then, of course, $\left[b_{1}, b_{2}, b_{3}\right]$ (that is, the plurality of the things identical to either $b_{1}, b_{2}$, or $b_{3}$ ) are also identical to $\left[\left[a_{1}, a_{2}\right],\left[a_{1}, a_{3}\right],\left[a_{2}, a_{3}\right]\right]$ (that is, to the plurality of the things identical to either $\left[a_{1}, a_{2}\right],\left[a_{1}, a_{3}\right]$, or $\left.\left[a_{2}, a_{3}\right]\right)$; if $a_{1}$ is part of $b_{1}$ and $b_{1}$ is identical to $\left[a_{1}, a_{2}\right]$, then, of course, $a_{1}$ is also a part of $\left[a_{1}, a_{2}\right]$.

It can also be straightforwardly proved from (TheP) that the plurality of the bricks is different from the plurality of the atoms:

$$
\left[b_{1}, b_{2}, b_{3}\right] \neq\left[a_{1}, a_{2}, a_{3}\right]
$$

In fact, none of the atoms is identical to a brick and none of the bricks is identical to an atom:

(19) $\sim \exists x\left(x \prec\left[a_{1}, a_{2}, a_{3}\right] \wedge\left(x=b_{1} \vee x=b_{2} \vee x=b_{3}\right)\right)$

(20) $\sim \exists x\left(x \prec\left[b_{1}, b_{2}, b_{3}\right] \wedge\left(x=a_{1} \vee x=a_{2} \vee x=a_{3}\right)\right)$

The idea that the plurality of the bricks is different from the plurality of the atoms might raise two worries: (i) How can the two pluralities be different given that each of the bricks is identical to a plurality of atoms? (ii) Isn't this result incompatible with the very intuition that lies behind SCAI-theories in general?

As for the first worry, the question boils down to what it means for two pluralities to be identical. In Sect. 1 we encountered the (One-of) principle, of which (One-of ${ }_{G}$ ) is the generic version. (One-of) says that identical pluralities have the same members. However, $\left(\mathrm{ACAI}_{\mathrm{C}}\right)$-theorists can simply claim that $\left(\right.$ One-of $\left._{\mathrm{G}}\right)$ follows from the very definition of plural identity ${ }^{45}$ :

\footnotetext{
44 See footnote 4.

45 Authors that define plural identity along these include van Inwagen (1994, p. 211), Yi (1999, p. 84, 2006, p. 243, 2014, p. 175), and Cotnoir (2014, p. 19), among others.
} 
(PI-def) $\quad x x=y y=d f \forall z(z \sqsubset x x \leftrightarrow z \sqsubset y y)$

According to (PI-def), what it is for the $x x$ and the $y y$ to be identical is to have the same members. Therefore, once (PI-def) is on board there should be no mystery as to why the plurality of the atoms and the plurality of the bricks are different: they contain different members. ${ }^{46}$

As for the second worry, the wall, the bricks, and the atoms can be said to be the same 'portion of reality' in virtue of having the same 'atomic footprint' (Loss 2019, p. 15). Letting ' $\mathbb{P}^{x}$, stand for 'the plurality of atoms $x$ is identical to', the notion of atomic footprint of a singular entity $x(' A F(x)$ ') and the notion of atomic footprint of a plurality $\left[x_{1}, \ldots, x_{n}\right]$ of entities (' $A F\left(\left[x_{1}, \ldots, x_{n}\right]\right)$ ') can be defined as follows (where ' $\alpha_{1} \cup \cdots \cup \alpha_{n}$ ' stands for the plurality containing, for each $\alpha_{m}(1 \leq m \leq n$ ), either $\alpha_{m}$, if $\alpha_{m}$ is an atom, or all the members of $\alpha_{m}$, if $\alpha_{m}$ is a plurality of entities, and nothing else):

$$
\begin{array}{ll}
(\mathrm{AF}(x)-\mathrm{def}) & \text { if } x \text { is a composite entity, } A F(x)={ }_{d f} \mathbb{P}^{x} \\
& \text { if } x \text { is an atom, } A F(x)=d f x \\
(\mathrm{AF}(x x) \text {-def }) & A F\left(\left[x_{1}, \ldots, x_{n}\right]\right)={ }_{d f} A F\left(x_{1}\right) \cup \ldots \cup A F\left(x_{n}\right)
\end{array}
$$

It can be easily checked that the following holds:

(21) $A F(c)=A F\left(\left[b_{1}, b_{2}, b_{3}\right]\right)=A F\left(\left[a_{1}, a_{2}, a_{3}\right]\right)$

Therefore, also $\left(\mathrm{ACAI}_{\mathrm{C}}\right)$ can straightforwardly account for the innocence of mereology. The wall is 'nothing over and above' both the atoms and the bricks because, in virtue of being identical to the atoms, the atomic footprint of the wall is identical to the atomic footprint of both the bricks and the atoms.

Notice, finally, that even if $\left(\mathrm{ACAI}_{\mathrm{C}}\right)$-theorists are committed to the existence of super-pluralities (in the sense specified above) they are not committed to the existence of super-super-pluralities, that is, pluralities having (proper) superpluralities as members. For instance, the wall $c$ is different from the super-plurality $\left[\left[a_{1}, a_{2}\right],\left[a_{1}, a_{3}\right],\left[a_{2}, a_{3}\right]\right]$. Therefore, one cannot consider a plurality having the wall $c$ and a different wall $d$ (having $\left[e_{1}, e_{2}, e_{3}\right]$ as its atomic footprint) as members and argue that the plurality $[c, d]$ is identical to the super-super-plurality $\left[\left[\left[a_{1}, a_{2}\right],\left[a_{1}, a_{3}\right],\left[a_{2}, a_{3}\right]\right],\left[\left[e_{1}, e_{2}\right],\left[e_{1}, e_{3}\right],\left[e_{2}, e_{3}\right]\right]\right]$. More in general, it only follows from $\left(\mathrm{ACAI}_{\mathrm{C}}\right)$ that the only pluralities that are identical to a singular entity are plurality of atoms, which are clearly not super-pluralities. Therefore, although it does follow from $\left(\mathrm{ACAI}_{\mathrm{C}}\right)$ (and the fact that there are composite entities) that there are pluralities containing pluralities of atoms, it doesn't follow that there are pluralities containing pluralities of pluralities of atoms.

\section{Conclusion}

According to Sider (2014) and I (Loss 2019), SCAI-theorists must uphold some weak form of the plural comprehension principle. As I have suggested, it is wrong to con-

\footnotetext{
46 The 'members' of the plurality of the bricks are such that they themselves contain some members. Notice, however, that this is not problematic in our case. In fact, within a framework that admits the existence of super-pluralities (in the sense specified above) it appears highly plausible not to take the one-of relation to be transitive.
} 
sider this option to be damning for SCAI-theorists. In fact, at least SCAI-theorists embracing (ACP) as their plural comprehension principle appear to be perfectly in position to claim that, while their theory is cast by means of the most joint-carving plural quantifier (which quantifies only over pluralities of fundamental entities), the standard 'unrestricted' plural comprehension principle (CMP) is expressed by means of a less joint-carving plural quantifier. Finally, as I have argued in Sect. 4, ACAItheorists appear to be forced to allow the existence of 'super-pluralities' in their theory, that is, pluralities having proper pluralities as 'members'. However, this seems to be a price that may be well worth paying in order to have both an innocent mereology and the full power of plural quantification. ${ }^{47}$

Acknowledgements Open Access funding provided by Projekt DEAL. I have presented parts of this paper at the workshop Identity, extensionality and constitution at the University of Leiden and at the Forschungskolloquim at the Philosophy department at the University of Hamburg. I am very grateful to the audiences in both occasions for their valuable feedback. I am also very grateful to three anonymous referees for providing insightful comments which greatly helped improve the paper.

Open Access This article is licensed under a Creative Commons Attribution 4.0 International License, which permits use, sharing, adaptation, distribution and reproduction in any medium or format, as long as you give appropriate credit to the original author(s) and the source, provide a link to the Creative Commons licence, and indicate if changes were made. The images or other third party material in this article are included in the article's Creative Commons licence, unless indicated otherwise in a credit line to the material. If material is not included in the article's Creative Commons licence and your intended use is not permitted by statutory regulation or exceeds the permitted use, you will need to obtain permission directly from the copyright holder. To view a copy of this licence, visit http://creativecommons.org/licenses/by/4.0/.

\section{References}

Baxter, D. (1988). Many-one identity. Philosophical Papers, 17(3), 193-216.

Bennett, K. (2015). Perfectly understood. In B. Loewer \& J. Schaffer (Eds.), unproblematic, and certain: Lewis on mereology. A companion to David Lewis (pp. 250-261). Hoboken: Wiley-Blackwell.

Bøhn, E. D. (2014). Unrestricted composition as identity. In D. Baxter \& A. Cotnoir (Eds.), Composition as identity (pp. 143-165). Oxford: Oxford University Press.

Bøhn, E. D. (2019). Composition as identity: Pushing forward. Synthese. https://doi.org/10.1007/s11229-0 19-02193-X.

Boolos, G. (1984). To be is to be a value of a variable (or to be some values of some variables). The Journal of Philosophy, 81(8), 430-449.

Boolos, G. (1985). Nominalist platonism. Philosophical Review, 94(3), 327-344.

Bricker, P. (2016). Composition as a kind of identity. Inquiry: An Interdisciplinary Journal of Philosophy, 59(3), 264-294.

Bricker, P. (2019). Composition as identity, Leibniz's law, and slice-sensitive emergent properties. Synthese. https://doi.org/10.1007/s11229-019-02106-y.

Calosi, C. (2016). Composition is identity and mereological nihilism. The Philosophical Quarterly, 263, 219-235.

\footnotetext{
47 Concerning the relation between Composition as Identity and higher-order plural quantification it is interesting to notice in his version of count-relative SCAI, Cotnoir (2013) employs a semantics according to which the denotation of a singular term is a set of atoms, while the denotation of a plural term is a set of sets of atoms. However, he claims that 'hyperplurals' could also be used instead of sets: 'For those with ontological qualms about using set theory for semantics, the semantics could be done using hyperplurals; but given the relative unfamiliarity with higher-order plural quantification, the set-theoretic interpretation will serve as a useful guide.' (Cotnoir 2013, p. 301).
} 
Calosi, C. (2018). Failure or boredom: the pendulum of composition as identity. American Philosophical Quarterly, 55(3), 281-292.

Cotnoir, A. (2013). Composition as general identity. In K. Bennett \& D. Zimmerman (Eds.), Oxford studies in metaphysics (Vol. 8, pp. 295-322). Oxford: Oxford University Press.

Cotnoir, A. (2014). Composition as identity: Framing the debate. In D. Baxter \& A. Cotnoir (Eds.), Composition as identity (pp. 3-23). Oxford: Oxford University Press.

Gruszczyński, R. (2015). On mereological counterparts of some principle[s] for sets. Logique et Analyse, 232, 535-546.

Hawley, K. (2014). Ontological innocence. In D. Baxter \& A. Cotnoir (Eds.), Composition as identity (pp. 70-89). Oxford: OUP.

Hazen, A. (1997). Relations in Lewis's framework without atoms. Analysis, 57(4), 243-248.

Hovda, P. (2009). What is classical mereology? Journal of Philosophical Logic, 38(1), 55-82.

Javier-Castellanos, A. (2019). Quantifier variance, ontological pluralism and ideal languages. Philosophical Quarterly, 69(275), 277-293.

Linnebo, Ø. (2017). Plural quantification. In E. Zalta (Ed.), The Stanford encyclopedia of philosophy (Summer 2017 Edition). Retrieved August 19, 2019, from https://plato.stanford.edu/archives/sum2017/ entries/plural-quant/.

Linnebo, Ø., \& Nicolas, D. (2008). Superplurals in English. Analysis, 68(3), 186-197.

Loss, R. (2016). Parts ground the whole and are identical to it. Australasian Journal of Philosophy, 94(3), 489-498.

Loss, R. (2018). A sudden collapse to nihilism. The Philosophical Quarterly, 68, 370-375.

Loss, R. (2019). On atomic composition as identity. Synthese. https://doi.org/10.1007/s11229-019-022956.

Loss, R. (2020). Correction to: On atomic composition as identity. Synthese. https://doi.org/10.1007/s112 29-020-02617-z.

McKay, T. (2006). Plural predication. Oxford: Oxford University Press.

Moltmann, F. (2016). Plural reference and reference to a plurality. Linguistic facts and semantic analyses. In M. Carrara, A. Arapinis, \& F. Moltmann (Eds.), Unity and plurality. Logic, philosophy, and semantics (pp. 93-120). Oxford: Oxford University Press.

Oliver, A., \& Smiley, T. (2013). Plural logic. Oxford: Oxford University Press.

Payton, J. D. (2019). How to identify wholes with their parts. Synthese. https://doi.org/10.1007/s11229-01 9-02191-z.

Rayo, A. (2006). Beyond plurals. In A. Rayo \& G. Uzquiano (Eds.), Absolute generality (pp. 220-254). Oxford: Oxford University Press.

Schaffer, J. (2010). Monism: The priority of the whole. Philosophical Review, 119, 31-76.

Sider, T. (2007). Parthood. The Philosophical Review, 116, 51-91.

Sider, T. (2011). Writing the book of the world. Oxford: Oxford University Press.

Sider, T. (2013). Against parthood. In K. Bennett \& D. Zimmerman (Eds.), Oxford studies in metaphysics (Vol. 8, pp. 237-293). Oxford: Oxford University Press.

Sider, T. (2014). Consequences of collapse. In D. Baxter \& A. Cotnoir (Eds.), Composition as identity (pp. 211-221). Oxford: Oxford University Press.

Simons, P. (2016). The ontology and logic of higher-order multitudes. In M. Carrara, A. Arapinis, \& F. Moltmann (Eds.), Unity and plurality: Logic, philosophy, and linguistics (pp. 55-69). Oxford: Oxford University Press.

van Inwagen, P. (1994). Composition as identity. Philosophical Perspectives, 8, 207-220.

Varzi, A. (2014). Counting and countenancing. In D. Baxter \& A. Cotnoir (Eds.), Composition as identity (pp. 70-89). Oxford: Oxford University Press.

Varzi, A. (2016). Mereology. In E. Zalta (Ed.), The Stanford encyclopedia of philosophy (Winter 2016 Edition). Retrieved August 19, 2019, from https://plato.stanford.edu/archives/win2016/entries/ mereology.

Varzi, A. (2017). On being ultimately composed of atoms. Philosophical Studies, 174(11), 2891-2900.

Wallace, M. (2011a). Composition as identity: Part 1. Philosophy Compass, 6(11), 804-816.

Wallace, M. (2011b). Composition as identity: Part 2. Philosophy Compass, 6(11), 817-827.

Yi, B. (1999). Is mereology ontologically innocent? Philosophical Studies, 93, 141-160. 
Yi, B. (2006). The logic and meaning of plurals, Part II. Journal of Philosophical Logic, 35(3), 239-288.

Yi, B. (2014). Is there a plural object? In Donal Baxter \& Aaron Cotnoir (Eds.), Composition as identity (pp. 169-191). Oxford: Oxford University Press.

Publisher's Note Springer Nature remains neutral with regard to jurisdictional claims in published maps and institutional affiliations. 